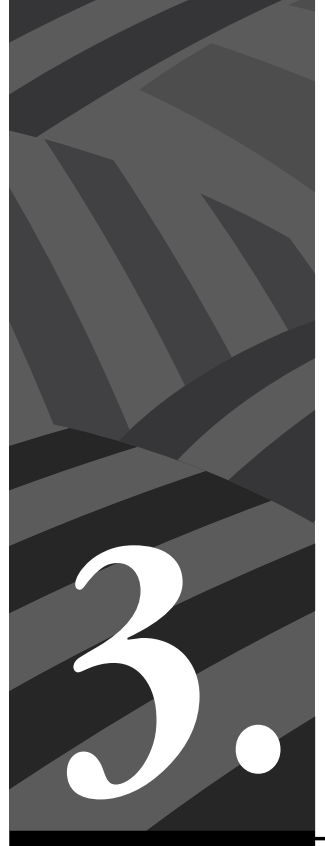

\title{
Interseccionalidad en el proceso de revelación y aceptación de la orientación sexual de hijos homosexuales en dos familias afrocolombianas
}




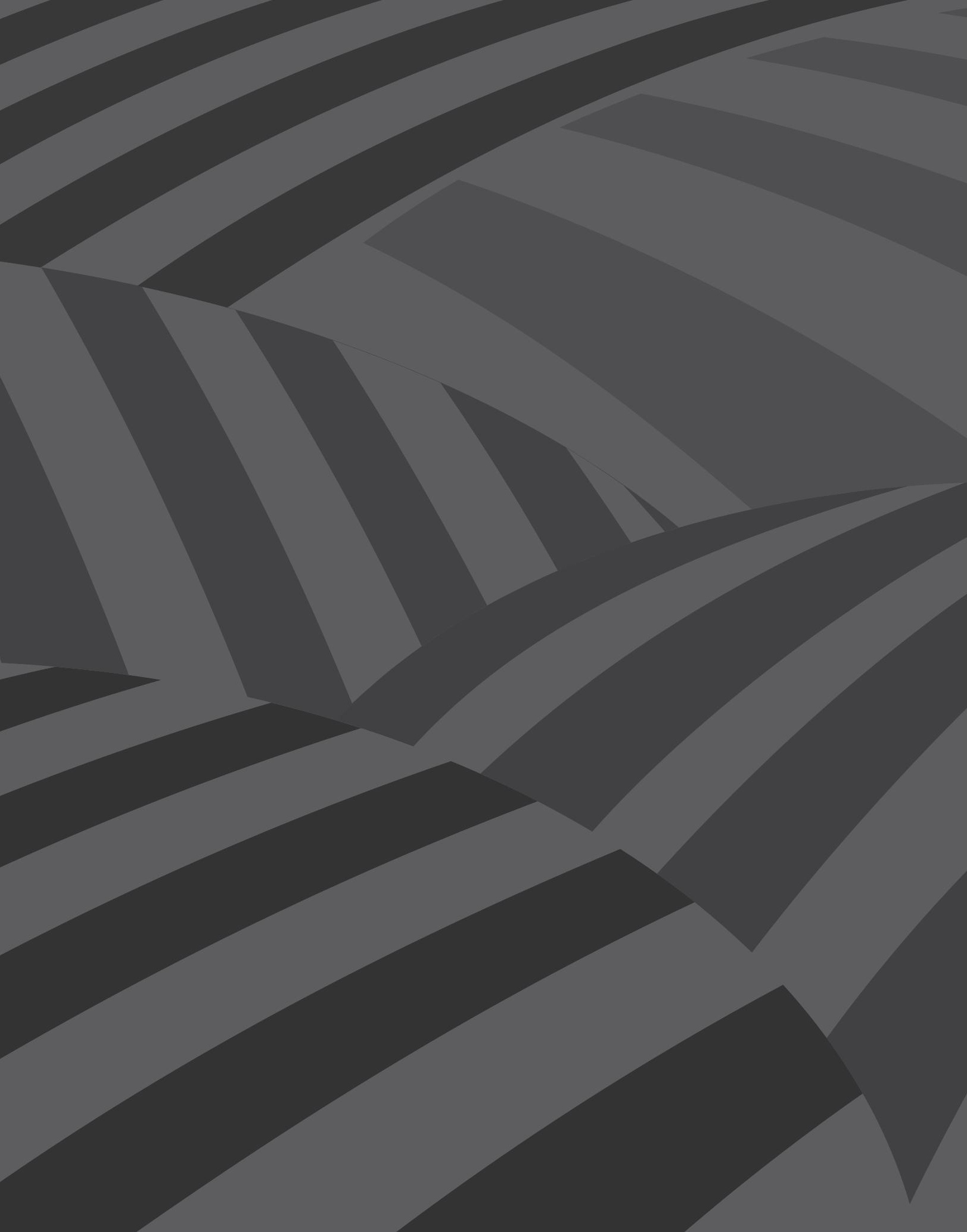




\title{
Interseccionalidad en el proceso de revelación y aceptación de la orientación sexual de hijos homosexuales en dos familias afrocolombianas"
}

\author{
Por Angélica María Galvis Rivas**, Jessica Natalia Gómez Rocha***, Linda Teresa Orcasita****, \\ Juan Sebastián Rueda-Toro*****
}

Resumen: En Colombia, la población negra, afrocolombiana, raizal y palenquera equivale a 4671 160 personas. Los datos de encuestas nacionales evidencian, en una muestra de 92799 personas, que el $0,4 \%$ de las mujeres y el $1,2 \%$ de los hombres se identifican como homosexuales. Ante esto, aparece el concepto de interseccionalidad que muestra relaciones existentes entre varias formas de discriminación, especialmente en la diversidad étnico-racial y sexo-genérica. Esta investigación cualitativa buscó comprender el proceso de revelación y aceptación de la orientación homosexual de los hijos en dos familias afrocolombianas, por medio de entrevistas realizadas a la diada madrehijo. Se identifican las características del proceso de revelación y aceptación, así como los recursos y barreras percibidas por las familias durante dicho proceso. Se evidencia la importancia de

* $\quad$ Este artículo se deriva de la investigación “Dinámicas familiares frente al proceso de revelación y aceptación de la orientación sexual de hijos gays e hijas lesbianas de la ciudad de Cali-Colombia" (Código 020100594), realizada entre el 1 de febrero y el 14 de diciembre de 2018 y financiada por la Oficina de Investigación y Desarrollo (OID) de la Pontificia Universidad Javeriana, Seccional Cali.

** Psicóloga, Pontificia Universidad Javeriana Cali, Colombia. Correo: angelicagalvisr@hotmail.com

*** Psicóloga, Pontificia Universidad Javeriana Cali, Colombia. Correo: jessica_gómez80@hotmail.com

**** Psicóloga y magíster en Familia, Pontificia Universidad Javeriana Cali, Colombia, Facultad de Humanidades y Ciencias Sociales. Docente Departamento de Ciencias Sociales. Directora del grupo de investigación Bienestar, Trabajo, Cultura y Sociedad (BITACUS) y la línea Familias, Género y Sexualidad. Correo: ltorcasita@javerianacali. edu.co. ORCID https://orcid.org/0000-0002-7599-9280. CVLAC: https://scienti.colciencias.gov.co/cvlac/visualizador/generarCurriculoCv.do?cod_rh $=0001001914$

***** Joven investigador Minciencias, Convocatoria 850, Pontificia Universidad Javeriana Cali,Colombia.GrupodeinvestigaciónBienestar,Trabajo,CulturaySociedad(BITACUS). Línea Familias, Género y Sexualidad. Correo: sebastianrueda@javerianacali.edu. co. ORCID https://orcid.org/0000-0003-3824-4310. CVLAC: https://scienti.colciencias.gov.co/cvlac/visualizador/generarCurriculoCv.do? cod_rh $=0000119330$ 
abordar la perspectiva de doble discriminación por etnia/raza y orientación sexual, así como el diseño de intervenciones que reconozcan el enfoque diferencial en la población.

Palabras clave: proceso de revelación y aceptación, hijos homosexuales, madres, comunidad afrocolombiana, interseccionalidad.

\section{Intersectionality in the Process of Revelation and Acceptance of Coming Out in Two Afrocolombian Families}

Abstract: In Colombia, the Black, Afro-Colombian, Raizal and Palenquera populations are equivalent to 4,671,160 people. The data from national surveys show in a sample of 92799 people, $0.4 \%$ of women and $1.2 \%$ of men identified as homosexual. Given this, the concept of intersectionality appears showing existing relationships between various forms of discrimination. This research sought to understand the process of coming out of two children from Afro-Colombian families with a qualitative study, with interviews with the children and their mothers. The characteristics of the disclosure and acceptance process and resources and barriers perceived by the families are identified. The importance of addressing the perspective of double discrimination based on ethnicity/race and sexual orientation and the design of interventions that recognize the differential approach in the population is evident.

Keywords: disclosure and acceptance process; gay children; mothers; afro-colombian community; intersectionality.

Cómo citar este artículo: Galvis Rivas, Angélica María; Gómez Rocha, Jessica Natalia; Orcasita, Linda Teresa; Rueda-Toro, Juan Sebastián (2020). Interseccionalidad en el proceso de revelación y aceptación de la orientación sexual de hijos homosexuales en dos familias afrocolombianas. Revista Controversia, 215, 75-108.

Fecha de recepción: 28 de marzo de 2020

Fecha de aprobación: 22 de junio de 2020

\section{Introducción}

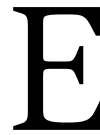

n el Censo Nacional de Población y Vivienda, del Departamento Administrativo Nacional de Estadística (DANE), año 2019, se estimó que en Colombia la población autorreconocida como negra, afrocolombiana, raizal y palenquera equivalía a 4671160 personas, lo cual representaba el 9,34\% del total de la población nacional. Sin 
embargo, en el documento Hacia una nación pluriétnica y multicultural 1998-2002, de la Comisión de Estudios para la Formulación del Plan de Desarrollo de las Comunidades Negras, se reconoce que esta población ascendía a 10,5 millones, calculados según un porcentaje variable aplicado a los municipios con comunidades negras (Programa de las Naciones Unidas para el Desarrollo (PNUD, 2011; Ministerio de Salud y Protección Social, 2015). Estas cifras evidencian la importancia de generar investigaciones relacionadas con la población afro ${ }^{1}$ del país, teniendo en cuenta las desigualdades sociales a las que esta se enfrenta, desde el punto de vista de estigmas, prejuicios y discriminación.

Las desigualdades sociales en Colombia y América Latina conforman la razón principal por la cual es prioritario abordar temas socio-raciales en las investigaciones y procesos de intervención. Las categorías raza, género, orientación sexual (os) y sexualidad van más allá de poder analizar las políticas de identidad y reconocimiento, y permiten también analizar las relaciones (Curiel, 2014).

La historia de las poblaciones indígenas y afrodescendientes está atravesada por años de esclavitud, sometimiento y discriminación, situaciones que propiciaron la conformación de grupos posesclavistas de gran número, en especial en Colombia (Curiel, 2014). Desde 1993, por medio de la Ley 70 del Congreso de la República, se reconoce en Colombia a las comunidades negras. Esta ley define a esta comunidad como el "conjunto de familias de ascendencia afrocolombiana que poseen una cultura propia, comparten una historia y tienen sus propias tradiciones y costumbres dentro de la relación campo-poblado, que revelan y conservan conciencia de identidad que las distinguen de otros grupos étnicos” (art. 2o, num. 5). En este caso se tiene en cuenta la importancia de la cultura,

1 En la literatura se refieren a la población afro de diferentes maneras, por lo que en aquellos fragmentos en donde se mencionan las ideas de otros investigadores/as se respetarán los términos utilizados por estos/as. Sin embargo, los autores/as de este estudio reconocen esta población como comunidad afrocolombiana y personas con os no heteronormativa. 
al ser esta la que media y establece las pautas requeridas para comprender cómo se construye y se expresa la sexualidad en su complejidad y con todos sus matices (Moncada-Santos y Fontelo-Danta, 2018).

Como resultado del proceso social en el que se evidenció la interrelación de las desigualdades sociales relacionadas con las categorías de sexo, raza, género y sexualidad, surge el concepto de interseccionalidad propuesto por un grupo de mujeres negras de Estados Unidos, iniciadoras del feminismo negro (Black Feminism). Así, el concepto de interseccionalidad muestra las relaciones existentes entre varias formas de discriminación y las vulnerabilidades que estas conllevan (Rubio, 2014). El reto actual de los estudios o movimientos que abordan este tema sería no categorizar las experiencias como exclusión de algún tipo de forma individual, sino analizar cómo estas dimensiones se cruzan e interactúan, basadas en la premisa de que los sistemas mayores de opresión se eslabonan ejerciendo opresiones múltiples sobre las comunidades afrocolombianas como proponen Cunin (2007) y Almario et al. (2014).

En relación con las desigualdades sociales, las comunidades afrocolombianas tienen presencia en territorios colectivos con mayor vulnerabilidad sociodemográfica, como el Pacífico nariñense y el Pacífico caucano, donde se registran las más altas tasas de analfabetismo, mayor tasa global de fecundidad y mayor índice de pobreza, miseria y hambre (PNUD, 2011).

Por otra parte, según la Encuesta Nacional de Demografía y Salud, del Ministerio de Salud y Profamilia (2015), realizada con una muestra de 92799 personas, de las cuales 52479 fueron mujeres de 13 a 69 años y 40300 hombres de 13 a 69 años, el 99\% de las mujeres manifestó ser heterosexual, el $0,4 \%$ homosexual y el $0,6 \%$ bisexual. En cuanto a los hombres, 98,2\% dijo ser heterosexual, 1,2\% homosexual y $0,6 \%$ bisexual. Esto aporta información importante sobre la población de lesbianas, gais, bisexuales, intersexuales y otras disidencias sexuales (LGBTI +) en Colombia, las cuales según Ortiz-Hernández (2004), 
históricamente han sido afectadas por situaciones de discriminación, violencia y exclusión.

Cabe resaltar que autores como Mogrovejo (2008), Salinas (2010) y González (2016) critican el concepto diversidad sexual y la sigla LBGT, y aseguran que estas categorías han perpetuado el control político de las sexualidades e identidades de género distintas de la heterosexual, retornando la academia a la dicotomía hombre-mujer y otorgando a cada letra una forma estática de ser, sentir y amar. Por esta razón, Salinas (2010) e Isaza-Camargo (2018) plantean el concepto disidencias sexuales, como una manera de posicionamiento político de resistencia a concepciones fijas de la identidad sexual y mecanismos de jerarquización sexual que reproducen formas de desigualdad social.

En relación con lo anterior, la reproducción de estereotipos en las familias no solo remite a la socialización de los sujetos en sociedades heteronormativas, sino a cómo estos experimentan las situaciones de estigma. El estigma, entendido como un proceso de interiorización que se evidencia en los modos en que estas personas construyen sus experiencias familiares como reflejo de los modelos de referencia heterosexual (Libson, 2012). Así, a pesar de las luchas que han emprendido movimientos feministas y LGBTI + , como los define Libson, aún siguen presentes diversos estereotipos en las transmisiones familiares, en el anclaje cultural de la "masculinidad hegemónica", como la menciona el autor, y en la sociedad patriarcal, la institucionalización de la heterosexualidad obligatoria y los lugares privilegiados asignados a las madres en relación con sus hijos.

El acto de revelar una os no hegemónica por parte de un/a joven puede ser un desencadenante de estrés, incluyendo las reacciones de las personas a las que se hacen las revelaciones (Rothman, Sullivan, Keyes y Boehmer, 2012). Es decir, el aspecto que mayor temor genera en los/las jóvenes respecto al proceso de revelación es la reacción de las personas pertenecientes a su contexto más próximo. Igualmente, es común que 
los/las jóvenes con una os disidente teman respuestas adversas y repercusiones negativas de sus padres (Willoughby, Malik y Lindahl, 2006). No obstante, dicho proceso puede llegar a generar bienestar físico y psicológico favoreciendo la resiliencia y el orgullo en los/las jóvenes, a partir de factores protectores como una autoestima alta y el apoyo familiar (Jiménez, Borrero y Nazario, 2011; Orcasita, et al., 2020).

Carrion y Lock (1997) proponen ocho etapas que permiten comprender las luchas internas por las que atraviesan los/as jóvenes desde la autorrevelación hasta la integración eventual de relaciones dinámicas y fluidas con la identidad homosexual. La primera etapa se denomina descubrimiento interno de la orientación sexual. En esta, se habla de cuatro conflictos emocionales: el desconcierto, la vergüenza, la minimización y la negación; estos abarcan sucesos de tipo cognitivo y solo un poco de carácter emocional; la persona experimenta miedo, rechazo y abandono ante la autopercepción de ser diferente, y en su intento de negar su os experimenta el gusto por ambos sexos. La segunda es la atracción del objeto sexual. Esta dependerá de los resultados obtenidos en la primera etapa, es decir, si la persona no logra resolver el conflicto de negación quedaría estancada en sentimientos y emociones negativas que desencadenarían malestar psicológico. La tercera etapa, aceptación temprana del yo sexual, hace referencia al inicio de la integración entre el yo, la os y la identidad; aquí la persona empieza a imaginar su vida y su futuro como homosexual (Rueda, 2015).

La cuarta etapa, congruencia de prueba, corresponde al momento en que la persona inicia su relacionamiento con personas que identifique se definen a sí mismas como homosexuales. En la quinta etapa, mayor aceptación del yo sexual integrado, la persona puede revelar su os siempre y cuando haya tenido éxito en las etapas anteriores. En esta etapa la persona inicia pensando positivamente acerca de sí misma, relacionándose sexualmente con personas de su mismo sexo y estableciendo vínculos más cercanos con personas homosexuales. La sexta etapa, consolidación de la autoestima, se caracteriza por la comparación entre 
la percepción interna y las percepciones sociales; en este momento la persona tiene la capacidad de tomar postura frente a lo que opina el contexto sobre la homosexualidad (Rueda, 2015).

En la séptima etapa, llamada formación madura de la autoidentidad integrada, la persona promueve el orgullo de la aceptación de la identidad homosexual y se identifica con un mayor grupo de personas, lo que influirá de manera positiva en la forma de verse a sí misma. De esta manera, el bienestar psicológico y subjetivo subyace al bienestar social. Finalmente, en la octava etapa, denominada autoidentidad integrada dentro de un contexto social, en el desarrollo de la os se da un enriquecimiento mutuo entre la sociedad y la persona (Rueda, 2015). Así, esta teoría ofrece un modelo explicativo del desarrollo de la os en etapas, tareas y conflictos por resolver durante el proceso de revelación, el cual se reconoció apropiado para ser aplicado a personas gais y lesbianas, sin dejar de lado la importancia de mantener una mirada dinámica y ecológica de este fenómeno, en la que se reconoce que el desarrollo psicosocial es influenciado por factores idiosincráticos de índole social, económico, político, cultural, entre otros, propios de cada contexto (Bronfenbrener, 1987).

Por otra parte, según Rodríguez (2005), la discriminación es una conducta construida cultural, sistemática y socialmente, de desprecio y desdén hacia una persona o grupo de personas, basada generalmente en un prejuicio negativo o un estigma relacionado con una desventaja inmerecida, y que de manera intencional o no puede dañar sus derechos y libertades fundamentales. Respecto a la población de interés para esta investigación, se ha evidenciado que las comunidades afrodescendientes se encuentran marginadas de los beneficios del desarrollo, con un bajo nivel de calidad de vida expresado en términos de pobreza, inequidad, violencias, discriminación y fragmentación del tejido social, siendo su estado uno de los más críticos en la totalidad de la población colombiana (Consejo Nacional de Política Económica y Social, 2002). 
De acuerdo con la Comisión Interamericana de Derechos Humanos (CIDH, 2015), existe información preocupante sobre los altos niveles de discriminación y violencia contra las personas LGBT afrodescendientes. En América, afirma la $\mathrm{CIDH}$, dicha información se ha limitado a países como Brasil, Colombia, Nicaragua y Estados Unidos. Tomando en cuenta esto, las personas de descendencia africana con os e identidades de género no hegemónicas pueden enfrentar actos de violencia y discriminación debido a su raza, etnia, género, sexo, os, identidad de género, color de piel y/o situación de pobreza.

Además, se ha reportado que la violencia hacia personas LGBT afrodescendientes también encuentra una característica en función del sexo, dado que esta cultura presenta un fenómeno de hipermasculinidad o machismo reforzado que, en el caso de los hombres homosexuales, ha generado discriminación al identificarse en ellos conductas afeminadas (Urrea, Reyes y Botero, 2008). En el caso de las mujeres, la CIDH (2015) considera que la vulnerabilidad es mayor y más preocupante, dado que son quienes han sufrido una discriminación histórica con base en su os y género, pobreza extrema y raza. Es así como las actitudes machistas de esta cultura pueden resultar en la limitación de acceso a la educación y trabajo de las mujeres lesbianas y hombres gais afrodescendientes, y perpetuar la subordinación del género femenino.

Rothman et al. (2012) realizaron un estudio en el que evaluaron la asociación entre la revelación de la os, el apoyo de los padres y los indicadores de riesgo de salud física y mental. En los resultados de las encuestas se evidenció que cinco de los factores de riesgo para la salud evaluados fueron significativamente mayores en los/as gais y lesbianas, en comparación con los/as heterosexuales. Se encontró que en personas con altos niveles de riesgo en sus condiciones de salud, el $73 \%$ reportaron no haber divulgado su os a su familia, y más de la mitad de quienes dijeron no haber tenido apoyo familiar al momento de la revelación fueron evaluados como más propensos a sufrir trastornos alimenticios y abuso de sustancias psicoactivas. Debido a esto, es 
necesario reconocer la importancia de las dinámicas familiares frente al proceso de revelación de la os, y cómo estas pueden influenciar la salud física y psicológica de los/as jóvenes gais y lesbianas.

Otro estudio realizado por Baiocco et al. (2014) permitió concluir que los hijos/as se muestran más inclinados a divulgar su os, en primer lugar, a su madre, lo que llevó a afirmar que una reacción negativa materna podría afectar fuertemente la autoestima del/la joven, y que creencias conservadoras o la participación religiosa de los padres llegan a ser un predictor de prejuicios que generan que sean más propensos a reaccionar de manera negativa ante la revelación de la os de sus hijos/as.

Troutman y Evans (2014) aseguran que los padres se enfrentan a un desafío significativo cuando sus hijos/as adolescentes comienzan a identificarse con una os no heteronormativa, lo que produce momentos de desordenamiento en los miembros de la familia. Los autores sostienen que algunos padres experimentan pánico, un sentimiento de pérdida profunda, soledad existencial y vergüenza, ante lo que plantean que existe escasez de recursos en los padres y madres para afrontar la revelación de la os de sus hijos/as.

La familia es abordada por Hernández (2017) como una construcción cultural de valores sociales, tradicionales, religiosos y políticos que, en gran medida, se encuentran forjados por la historia. Estas características forman los estilos de relación interna entre los miembros de la familia, que interpretan valores brindados por la sociedad, tomando de ellos lo que consideran adecuado para su funcionamiento, concretándolo según su experiencia (Macionis y Plummer, 2007).

A partir de la revisión bibliográfica se pudo evidenciar que los temas de esta investigación han sido estudiados de manera aislada, es decir, se encuentran publicaciones sobre la revelación de la os de los/as jóvenes a su familia (Carrion y Lock, 1997; Baiocco et al., 2014; Rothman et al., 2012; Troutman y Evans, 2014) y existen estudios étnicos en los 
cuales ha sido relevante el componente cultural como objeto de estudio (Urrea et al., 2008), pero son pocos los trabajos que tienen en cuenta ambas dimensiones para brindar una explicación del fenómeno desde la interseccionalidad. Con base en lo expuesto, la investigación de la que se deriva este artículo buscó comprender el proceso de revelación y aceptación de la os en dos familias afrocolombianas, a partir de (1) identificar las características del proceso de revelación y aceptación, y (2) reconocer los recursos y barreras percibidos por cada una de las familias en el proceso.

\section{Método}

\section{Tipo y diseño de investigación}

La investigación fue de tipo cualitativo, con un diseño de estudio de caso, ya que se buscó abarcar la complejidad de dos situaciones particulares, pretendiendo la comprensión profunda de estas (Stake, 2007). Teniendo en cuenta que en los estudios cualitativos se favorece la complementariedad de métodos, se tuvo en cuenta la perspectiva narrativa en las experiencias de las dos familias, para encontrar aspectos comunes entre las historias de los participantes y nutrir las teorías en desarrollo ante un tema del cual se reporta poca bibliografía (Blanco, 2015; Salgado, 2007).

\section{Participantes}

Se contó con la participación de dos familias. La primera estuvo representada por una madre de 56 años (Teresa) y su hijo de 25 (Misael), que se autodenominó gay, ambos con creencias religiosas católicas. La segunda, por una madre de 51 años (María), católica, y su hijo (Johan) que se autodenominó como un hombre trans homosexual. ${ }^{2} \mathrm{El}$ tipo de muestreo fue no probabilístico por bola de nieve, que se refiere

2 Es importante reconocer que el estudio se focalizó en la orientación sexual y no en la identidad de género, aspecto fundamental para próximos estudios que aborden diversidad sexual y de géneros. 
al acceso a los participantes por medio de informantes clave conocidos previamente por los investigadores (Martínez-Salgado, 2012). Es importante mencionar que los padres no quisieron participar en el estudio, elemento que será importante en el análisis de los resultados.

\section{Instrumentos}

Se utilizaron dos herramientas de recolección de datos: una entrevista a profundidad (Taylor y Bogdan, 1992) y una línea de tiempo denominada "Mi viaje" (ver figura 1), construida en el proyecto macro al que se articula este estudio. La línea de tiempo cuenta con ilustraciones que permiten el abordaje de los diferentes momentos por los que puede atravesar una familia durante el proceso de revelación y aceptación de la os de un hijo/a. Los participantes pudieron elegir, de entre una serie de ilustraciones, cuáles fueron sus sentimientos, reacciones y decisiones en determinados momentos del proceso. Para la elaboración de las guías de entrevista se construyeron dos categorías de análisis que permitieron obtener la información necesaria para responder a los objetivos de la investigación:

(1) Proceso de revelación y aceptación, entendido como la situación donde se expresa de forma explícita y confirmatoria la os por parte de hijos gais e hijas lesbianas a sus familias (Vargas et al., 2011; Baiocco et al., 2014; Ceballos-Fernández, 2014; Rueda, 2015; Hernández, 2017).

(2) Recursos y barreras percibidos por familias afrocolombianas, que son patrones de conducta reforzados culturalmente en relación con la sexualidad, en los cuales se atribuyen estereotipos de la cultura afrocolombiana, y la disidencia sexual puede alterar el orden ideológico y desencadenar acciones de rechazo o facilitar el proceso de adaptación social, tomando las características de "ser negro" como un grado de superioridad ante otras disidencias sexuales (Gil, 2008; Urrea et al., 2008).

Ambos instrumentos fueron validados por cuatro jueces expertos y por medio de dos pruebas piloto. 
Figura 1. Técnica interactiva línea de tiempo "Mi viaje".

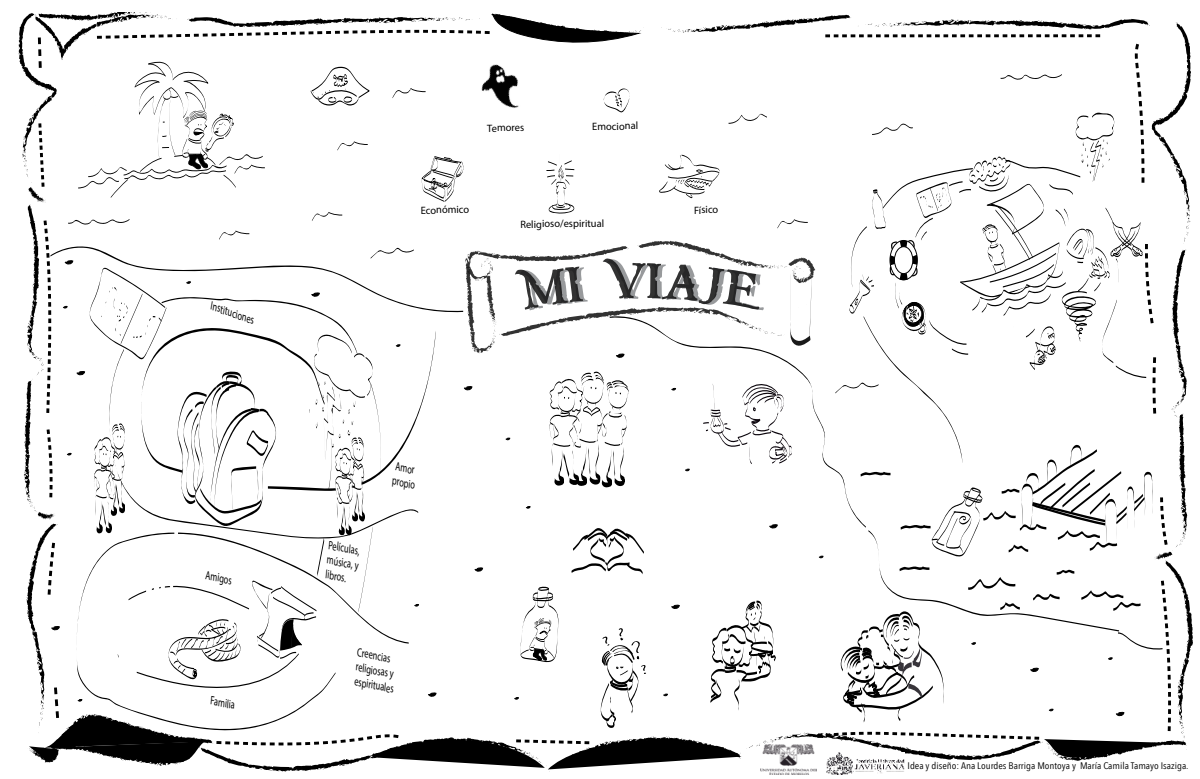

Fuente: investigación “Dinámicas familiares en el proceso de revelación y aceptación de la orientación sexual de hijos gays e hijas lesbianas en Cali-Colombia” (código: 020100594).

\section{Procedimiento}

La investigación se llevó a cabo en cuatro fases. En la primera, se contactó a la población. En la segunda, se recolectaron y sistematizaron los datos, aplicando las entrevistas y líneas de tiempo. En la tercera, se hizo el análisis temático de los datos (Mieles, Tonon y Alvarado, 2012) teniendo como temas principales las características del proceso de revelación, la vivencia de la familia en el proceso de aceptación de la os del hijo, y los recursos y barreras percibidos de la cultura afrocolombiana. Con esto se realizó un mapa de análisis de relaciones entre la información encontrada. En la cuarta y última fase, se hizo la socialización de resultados. 


\section{Consideraciones éticas}

Se buscó propiciar el respeto, dignidad, integridad y bienestar de las familias participantes, ejerciendo la confidencialidad de sus datos y el anonimato de sus respuestas. Igualmente, se contó con un consentimiento informado por parte de cada participante, donde se consignó el equipo investigador, los objetivos y riesgos del estudio. Finalmente, se hizo uso de pseudónimos para propiciar los derechos estipulados en la Resolución 8430 de 1993, del Ministerio de Salud de Colombia, para las investigaciones en salud con seres humanos, y la Ley 1090 de 2006, del Congreso de la República, para la investigación en psicología.

\section{Resultados}

Iniciando, se describe cada una de las familias, indicando sus principales datos sociodemográficos y características específicas de estructura y funcionamiento. Luego, se presentan los resultados más relevantes del proceso de revelación y aceptación vivido por cada uno de los/as participantes, para terminar con la descripción de los recursos y barreras percibidos, haciendo énfasis en aspectos propios de la cultura afrocolombiana. Cabe resaltar, nuevamente, que el acceso a cada familia se hizo por medio de entrevistas realizadas al hijo y a la madre, dado que los padres (hombres) no quisieron participar en el estudio.

\section{Las familias participantes}

Familia 1 (Misael de 25 años y Teresa de 56): conformada por cinco personas, cuatro hombres, y una mujer. Los padres estaban casados y vivían en un estrato socioeconómico dos; el hijo participante vivía con su pareja. La madre es procedente de Tumaco. Nivel educativo de la madre: primaria completa; nivel educativo del hijo participante: pregrado.

Familia 2 (Johan de 23 años y María de 51 años): conformada por cuatro personas, dos hombres y dos mujeres. El esposo actual de María no era el padre de sus hijos, pero convivía con ellos desde 17 años atrás. 
La familia vivía en un estrato socioeconómico dos. Johan nació en su ciudad de residencia y María provenía de un pueblo de Nariño, donde vivió hasta sus 9 años cuando se trasladó a Cali. Nivel educativo de la madre: secundaria incompleta; nivel educativo de Johan: estudios técnicos.

\section{Proceso de revelación y aceptación de la orientación sexual}

En las familias entrevistadas se evidenció que sus dinámicas familiares, contextos y situaciones de vida diferenciaban la vivencia de la revelación y aceptación de la os. En un primer momento, ambas madres coincidieron en no haber reconocido desde la infancia de sus hijos indicios que les permitieran sospechar sobre la os que más tarde les revelarían. En palabras de Teresa:

"Nunca sospeché, porque como uno tampoco se la pasaba mucho en la casa y uno llegaba a hacer las tareas (...) entonces nunca lo pensé"

(Teresa, 56 años, madre de Misael).

Los procesos de los participantes tienen aspectos diferenciadores, producto del tiempo que pasó desde el momento de la revelación hasta el día de la entrevista. Por ejemplo, Johan recordó así la edad en la que reveló a su madre que le gustaban las mujeres:

"Tenía 18 años... Me sentí bien porque me quité un peso de encima" (Johan, 23 años, hijo de María).

Misael, por su parte, tuvo un proceso con varios momentos de revelación. En primer lugar, su padre se dio cuenta por una persona externa al hogar. Posteriormente, Misael mismo le reveló a su madre su condición. Después, pasó un tiempo en que compartió con una pareja del sexo contrario, buscando cumplir con las expectativas de su familia y, finalmente, aceptó su os y la reiteró a su núcleo familiar. Por esta razón, Misael duplicó en su línea del tiempo una botella que representaba la revelación, haciendo alusión a los diferentes momentos de su proceso (ver figura 2). 
Figura 2. Representación de Misael sobre el momento de revelación.

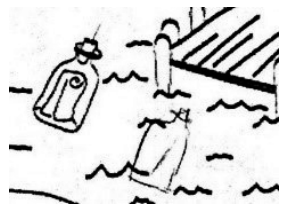

Fuente: fotografía tomada después de la entrevista.

Respecto a la primera persona escogida de su familia para revelar su os, Johan y Misael concordaron en que seleccionaron a su madre por el nivel de confianza que tenían con ella y por considerarla la persona más importante en su vida. En palabras de Johan:

"Es que cuando yo le conté, a la primera que se lo conté fue a mi mamá (...) pero en sí los primeros en saberlo fueron los de la calle (...) Porque en mi vida ella es la que, por decir algo "manda" (...)"

(Johan, 23 años, hijo de María).

Desde la perspectiva de las madres, ambas coincidieron en que el momento fue algo inesperado y que su reacción inicial fue no decir nada. Esto puede relacionarse con lo manifestado inicialmente respecto a que no tenían indicios, por lo que podría decirse que no hubo una preparación previa a la revelación.

En cuanto a la narración del hijo frente al proceso de formación de su identidad, tanto Misael como Johan expresaron que su os se comenzó a gestar desde la infancia, principalmente con el reconocimiento de que sus gustos eran diferentes a lo heteronormativo. Sin embargo, estas sensaciones y cuestionamientos las conservaron para sí mismos durante mucho tiempo. En palabras de Johan:

"En sí no fue un proceso, porque desde los cinco años yo me sentí pues, o sea no me fijé en un hombre, me fijé en una niña, desde ese entonces sé que no me gustan los hombres (...) cuando me gustó esa niña tenía como ocho o siete años (...)" (Johan, 23 años, hijo de María). 
Las narraciones de los hijos participantes tienen dos aspectos diferenciadores importantes para comprender su proceso y relatos de vida. Por un lado, las parejas que tuvo Misael le ayudaron en la formación de su identidad y en la preparación para el momento de revelar la situación a su familia. Cabe aclarar que sus parejas eran mayores que él y algunos ya habían revelado su os, por lo que la experiencia de otros fue fundamental para él:

“(...) claro, porque el hecho de yo compartir con mis parejas me hizo ver las inseguridades y los errores que yo estaba cometiendo frente a mí, entonces

para yo poder vivir con ellos me tocó enfrentar eso, y al irlo enfrentando empecé a darme cuenta de lo que tenía que hacer yo para poder salir de eso"

(Misael, 25 años, hijo de Teresa)

Por su parte, Johan tuvo un momento en que reveló a su madre ser lesbiana, pero con el paso del tiempo reconoció que se identificaba con una identidad masculina, por lo que al momento de la entrevista se definió como un hombre transgénero, pero aún no se sentía preparado para revelarlo a su familia. En sus palabras:

"Mm... pues haber es que no sé... yo como todavía no salgo, o sea no se lo he dicho a todo el mundo entonces, pero en sí soy una persona trans, no me gusta que me traten en femenino y a todos los que conozco pues les digo el nombre que me gusta que me digan y me visto con la ropa de hombres"

(Johan, 23 años, hijo de María).

Tanto Misael como Johan mencionaron que su principal expectativa frente a la revelación fue recibir apoyo por parte de su familia:

"Pues... no sé, el hecho de sentir apoyo (...)" (Johan, 23 años, hijo de María).

“(...) de pronto tener una persona en ese momento que me orientara de cómo poder manejar esa situación para no haber sentido todo lo que sentí en ese momento" (Misael, 25 años, hijo de Teresa). 
Un aspecto diferenciador fue la respuesta de los hijos participantes sobre cómo debería ser un proceso de revelación y aceptación ideal. Por un lado, Johan centró su discurso en que debía evitarse la discriminación, pues esto puede generar sentimientos negativos en la persona que está revelando. Por su parte, Misael reflexionó sobre el papel de los hijos como personas que deben comprender que para los padres no es un momento fácil de afrontar, por la cultura en la que se encuentran inmersos. En palabras de Johan:

"No sé, es que para mí pensar si uno siente apoyo antes de decir las cosas es más fácil, en cambio si uno siente o tiene la duda de que no van a aceptar o que lo van a discriminar de alguna forma o que va a perder tal cosa por el simple hecho de decirlo, uno se llena de más miedo y por eso hay personas

que no lo dicen" (Johan, 23 años, hijo de María).

En cuanto a los sentimientos experimentados frente a la revelación, las madres expresaron sentirse "enfrascadas", utilizando este término por la botella encontrada en la línea de tiempo. En el caso de María, mencionó que el momento fue difícil no solo porque ella aspiraba a cosas diferentes para su hijo, sino por la situación de drogadicción en la que se encontraban sus otros hijos y por los problemas de pareja que atravesaba, evidenciando así que contaba con estresores adicionales, en un contexto de vulnerabilidad que tuvo influencia en el proceso. En el caso de Teresa, el hecho de que ella quisiera aceptar a su hijo, pero a su esposo se le dificultara, hizo que fuera más difícil para ella.

Los principales temores frente al proceso de revelación y aceptación por parte de las madres se centraron en la preocupación por la seguridad de sus hijos, debido a la discriminación que pudieran sufrir y el temor por las infecciones de transmisión sexual asociadas a la homosexualidad. Adicionalmente, Teresa incluyó el aspecto religioso, pues para ella la Biblia le transmitía que debía rechazar a su hijo por su os, pero esto iba en contra de su amor por él. Ambos jóvenes coincidieron en que 
Figura 3. Representación de María sobre los sentimientos experimentados.

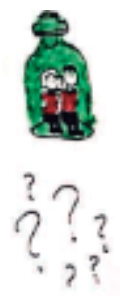

Fuente: fotografía tomada después de la entrevista.

su temor fue emocional, referente al sufrimiento que podía causarles el rechazo de su familia y de la sociedad, dado que reconocían que la homosexualidad aún es un tema difícil de aceptar para muchas personas; otro de sus temores consistió en imaginar de forma negativa cómo sería su futuro después de la revelación.

Dentro de los recursos identificados por las madres como facilitadores del proceso de revelación, surgió la imagen de Dios como guía para comprender el proceso, así como el amor hacia sus hijos. Respecto a los recursos identificados por los hijos, ambos coincidieron en el apoyo social. Para Johan fueron importantes sus compañeros de colegio, quienes le permitieron forjar su personalidad y dejar de lado su timidez para revelar su homosexualidad, mientras que para Misael fueron importantes tres exparejas que le ayudaron a apropiarse de su identidad y a tener la confianza suficiente para revelarla.

En cuanto a las barreras, María expresó la falta de redes de apoyo para afrontar la situación:

“(...) yo hubiera tenido una mamá, una hermana, un papá o me hubiera criado con una familia, de pronto hubiera sido más llevadera la situación (...)” (María, 51 años, madre de Johan) 
Los hijos coincidieron en que una barrera fue el temor a la discriminación social, dadas las actitudes de rechazo de la sociedad hacia las personas con os no heterosexual. Según Misael, esto se debía a las crianzas arraigadas y poco informativas frente a la diversidad sexual.

Finalmente, para Johan una barrera era la personalidad de su madre; Misael, por su parte, consideraba que la religión que su madre le había inculcado desde la infancia lo había ayudado en algunos aspectos, pero había sido barrera en otros.

\section{Recursos y barreras propios de la comunidad afrocolombiana}

En relación con los recursos culturales, ninguno de los miembros percibía elementos de este tipo que facilitaran el proceso. Con respecto a las barreras percibidas, desde sus diversas formas de expresarse todos los participantes identificaron elementos importantes que habían obstaculizado, bien el proceso de formación de su orientación homosexual (en el caso de los hijos), bien el manejo de las creencias culturales que causaban temores a las madres.

Johan narró hechos externos a su experiencia que consideraba como barreras de la cultura afrocolombiana frente al proceso de revelación; sin embargo, en ningún momento expresó que dichas situaciones le hubiesen sucedido a él, a diferencia de Misael, quien relató una situación de su infancia en la que la cultura fue una barrera para la expresión de su sexualidad. Desde las narrativas de ambos hijos:

"Pues en general, los que también son afros que no pertenecen a la familia de esa persona lo ven mal y dicen que no sé qué la misma sangre o que afrodescendientes cómo pueden salir, o sea, es como una deshonra para ellos, por así decirlo... Porque eso lo escuché una vez de una señora" (Johan, 23 años, hijo de María). 
“(...) pues mira que un compañero tenía una palabra en el colegio y una vez me lo dijo, siempre dicen que tras de negro feo y marica, tiene todas completas, tiene todos los males juntos. Siempre decía esa palabra supuestamente, pero entonces da a entender que el hecho de que usted sea moreno ya es malo de entrada, si es feo es malo y gay, entonces categorizado que eso es malo porque en la piel morena ha sido un esclavo, entonces está por debajo de la piel blanca (...)" (Misael, 25 años, hijo de Teresa).

Finalmente, ambas madres consideraron que la cultura afrocolombiana juzga a las personas con os diversas de forma más fuerte; las dos afirmaron que haber migrado desde edades tempranas de sus culturas de origen les permitió que su forma de ver el mundo cambiara, asegurando que podían aceptar la os de sus hijos gracias a que no vivían en sus lugares de procedencia. En palabras de María:

"Pues los afros, usted sabe que la cultura de los afros es como más, un marica como es "ay", puta, negro y marica ese negro, o sea nosotros los afros somos más machistas (...) o sea la sociedad lo ve mal visto, pero entre la comunidad

afro es peor todavía"

(María, 51 años, madre de Johan).

\section{Discusión}

Aquí se presenta el análisis de los resultados obtenidos con las dos familias afrocolombianas participantes en la investigación, partiendo del objetivo de comprender el proceso de revelación y aceptación de la os de sus hijos. Finalmente, se exponen las conclusiones y recomendaciones respecto al tema estudiado.

En relación con las características del proceso de revelación y aceptación, se identificó la importancia de la autoaceptación como un elemento fundamental para decidir revelar la os, lo que se relaciona con lo presentado por Rueda (2015) sobre la quinta etapa del modelo teórico de Carrion y Lock (1997) denominada mayor aceptación del yo sexual 
integrado, en la cual las personas revelan su os siempre y cuando hayan tenido éxito en las etapas del descubrimiento interno y de autoaceptación del yo sexual. La autoaceptación es fundamental en el proceso, dado que los hijos necesitan comprender y aceptar su os para integrarla a su identidad, expresarla a otras personas y estar preparados para las respuestas que puedan recibir.

Frente a los modelos teóricos sobre la formación de la identidad de las personas con os no hegemónica (Rueda, 2015), se confirma que no se siguen etapas lineales, sino que cada persona puede saltar de una etapa a otra de acuerdo con su historia de vida y contexto particular. Además, se reconoce la posibilidad de no vivir las etapas establecidas según la literatura, como lo demuestran los dos hijos participantes, quienes aunque tuvieron puntos comunes en su proceso — como haber revelado su homosexualidad primero a su madre, haber percibido su os desde la infancia y haber experimentado las mismas expectativas frente a las dificultades que tendrían que enfrentar después de revelar su os-, también tuvieron diferencias importantes como la edad, forma y momento de revelación, las experiencias previas que favorecieron su decisión de revelar y las barreras para hacerlo.

En cuanto a la manera como los hijos participantes identificaron que su os era diferente a la heterosexual, en ambos el descubrimiento se dio por la comprensión de que su os era diferente a lo enseñado por sus padres y lo observado socialmente; este proceso los llevó a experimentar miedo e incertidumbre frente a la posibilidad de ser rechazados (Carrion y Lock, 1997; Rueda, 2015; Willoughby et al., 2006).

Así, la primera etapa del modelo de Carrion y Lock (1997), denominada descubrimiento interno de la orientación sexual, se relaciona con lo identificado en los participantes, puesto que en esta se habla de cuatro conflictos emocionales (desconcierto, vergüenza, minimización y negación) que llevan a que la persona sienta miedo, rechazo y abandono, y a que en su intento de negar su os experimente gusto por hombres y mujeres. 
Ambos participantes mencionaron haber tenido temores emocionales derivados del miedo al rechazo de sus familias y de la sociedad, y de creer que su futuro sería negativo si revelaban su os; debido a esto, uno de ellos tuvo una pareja del sexo contrario intentando cambiar su os.

Ambos participantes expresaron que la persona a la cual revelaron primero su homosexualidad fue a su madre, por el rol importante que ella cumplía en su vida y la confianza que le tenían. Según Baiocco et al. (2014), Lujan y Tamarit (2012) y Vargas et al. (2011), los hijos/as se muestran inclinados/as a divulgar su os primero a su madre, lo que los llevó a afirmar que una reacción negativa materna podría afectar la autoestima del joven, aspecto que resulta importante para esta investigación, dado que en el caso de ambos participantes, aunque otras personas de su entorno ya sabían de su os o lo suponían, resaltaron la importancia de que su madre fuera la primera en conocer la situación a través de sus propias palabras.

Frente a los sentimientos de las dos madres participantes, ambas dijeron que tras la revelación de su hijo se sintieron "enfrascadas", término asociado a lo representado en la línea de tiempo, y que es entendido como incertidumbre, miedo y negación, sentimientos asociados al hecho de que su hijo no encajara en las expectativas que tenían, lo que se relaciona con lo señalado por Ceballos-Fernández (2014), quien menciona que existe un conjunto de esquemas, creencias y percepciones en las familias, que permean la crianza de los hijos/as, creando supuestos de deseabilidad en los padres, que se rompen cuando un hijo expresa su homosexualidad. No obstante, ambas madres señalaron que sus sentimientos se vieron influenciados por factores distintos a la os de su hijo, como problemas relacionados con sus otros hijos o su pareja y con el hecho de que al padre se le dificultara más aceptar la os.

Adicionalmente, ambas madres manifestaron un sentimiento de ambivalencia, ya que mientras expresaban aceptar a su hijo basadas en el amor y su creencia en Dios que les daba fuerza para sobrellevar la 
situación, mencionaban tener la esperanza de que cambiaran algún día y que su os fuera solo una etapa. Esto se relaciona con lo planteado por Vargas et al. (2011) respecto a que suelen presentarse reacciones ambivalentes, en las cuales los padres expresan apoyo y respeto ante la os de sus hijos/as, pero aseguran que la situación no puede hacerse pública, sino que debe mantenerse al interior de la familia, lo que Svab (2016) denomina closet familiar. De esta forma, la aceptación de las madres participantes podría verse como una ambivalencia, por la contradicción al expresar que se acepta la os de su hijo pero es mejor que esta se mantenga oculta.

Las barreras en el proceso de revelación y aceptación tuvieron que ver con la falta de redes de apoyo, la discriminación y el rechazo social, la crianza heteronormativa y la falta de información sobre diversidad sexual. Esto refuerza los planteamientos frente a la importancia de factores que contribuyan al bienestar de las personas LGBTI + mencionados por Orcasita et al. (2020), como el apoyo social familiar, y Zambrano, Ceballos y Ojeda (2017) sobre la educación en diversidad sexual dirigida a las familias.

En relación con los recursos percibidos en el proceso, ambas madres coincidieron en que la religión y el amor por su hijo fueron su principal apoyo. Por su parte, los hijos se refirieron a recursos sociales como la relación con compañeros de colegio y exparejas, lo que corrobora nuevamente la importancia del apoyo social durante el proceso de revelación y aceptación de la os en jóvenes gais y lesbianas (Orcasita et al., 2020; Vargas et al., 2011).

Tanto las madres como los hijos participantes dijeron sentirse orgullosas/os respecto a lo difícil que había sido el proceso y cómo habían ido avanzado. Sin embargo, se destaca que algunos no consideraron que el involucramiento familiar fuera impulsador del bienestar obtenido, sino la vivencia personal. Dicho reconocimiento resulta de suma importancia, dado que la postura que asuma la familia frente a la revelación de la 
os de alguno de sus miembros puede obstaculizar o beneficiar el desarrollo de la identidad de la persona y por lo tanto el bienestar de esta (Ceballos-Fernández, 2014).

Se identificó, también, que para los participantes, el pertenecer a la comunidad afrocolombiana no les aportó ningún beneficio en el proceso, por el contrario, les generó barreras. Esto, de acuerdo con Colombia Diversa (2018), es llamado endodiscriminación o discriminación hacia adentro, la cual hace referencia a los estigmas y actos discriminatorios de los movimientos sociales y comunidades que velan por los derechos de comunidades afro, en las que se rechaza que sus miembros se reconozcan como gais o lesbianas. Tal concepto concuerda con lo expresado por las madres participantes en este estudio, quienes si bien dijeron haber aceptado la os de su hijo, aclararon que lo hicieron en gran medida distanciándose de la comunidad afrocolombiana, y señalaron especialmente la ventaja de encontrarse lejos de su lugar de origen.

En relación con lo anterior, Urrea et al. (2008) afirman que se han creado patrones que refuerzan culturalmente ideas específicas sobre la expresión de la sexualidad en las comunidades afrocolombianas, entendidos como la racialización de la sexualidad, en la cual se da una atribución de estereotipos a determinada raza, que históricamente se han naturalizado; un ejemplo de esto es la exclusión que han sufrido hombres homosexuales negros tras reconocer su os o mostrarse "femeninos”, pues según lo expuesto, estas personas dejan de ser negros al perder el "atributo esencial" de una heterosexualidad patriarcal, lo que fue expresado tanto por los hijos como por las madres participantes, al puntualizar que si para la sociedad el ser gay o lesbiana sigue siendo motivo de discriminación, al interior de las comunidades afrocolombianas “es peor todavía”.

Lo expresado por las madres e hijos participantes coincide con lo planteado por Macionis y Plummer (2007), quienes afirman que existen valores y creencias que establecen lo que está bien y lo que está mal, 
los cuales varían dependiendo de las culturas y creencias ante las que las personas adoptan una postura. Según Urrea et al. (2008), vivir la sexualidad por fuera de la norma (entendida como lo típico o común en una cultura determinada), altera un orden ideológico, que en el caso de los hijos participantes de este estudio generó discriminación al ser parte de comunidades afrocolombianas y ser homosexuales, y los llevó a manifestar que la cultura afrocolombiana presenta esquemas sobre la sexualidad y su expresión que influyen en el comportamiento esperado por las personas, y representa una barrera en los procesos de aceptación y revelación de la os.

Respecto a lo anterior, se retoma el concepto de interseccionalidad (Cunin, 2007; Curiel, 2014) en la discriminación que expresaron haber vivido los hijos participantes frente al marcador de ser "negro" y ser "marica”, relacionando el primero con un nuevo hecho estigmatizante, esta vez de carácter histórico y temporal: la esclavitud, lo que podría verse como una reafirmación de la posición de inferioridad que ha sido atribuida a los afrocolombianos, sumada dicha intersección a la de personas homosexuales.

Cabe resaltar la evidencia de no coherencia entre género y orientación sexual que señaló Johan, pues aunque el análisis de la investigación estuvo centrado en el proceso de revelación que este había tenido cinco años antes como mujer lesbiana, su relato dio cuenta de que se había replanteado también su identidad de género, lo que es entendido por Vargas et al. (2011) como una transformación en la cual surgen nuevas necesidades referentes a la identidad, que pueden conllevar una nueva situación de crisis. Además, implica una nueva autovaloración y autoaceptación del yo respecto a los sistemas valorativos de la sociedad y un nuevo estigma para asumir, desde la intersección, el género. Johan, posterior a su revelación como mujer lesbiana, descubrió que no se identificaba con el género asignado al nacer y los estereotipos asociados a este; el día de la entrevista declaró identificarse con el género masculino y considerarse un hombre transgénero, con una os 
homosexual. Sin embargo, señaló que esto implicaría una nueva revelación a sus padres, para la que dijo no estar preparado, lo que podría relacionarse con realidades que demuestran que las personas negras y homosexuales se ven enfrentadas a una intersección de discriminaciones que las hace una población con gran vulnerabilidad (Castellanos, 2008; Rubio, 2014).

En conclusión, en primer lugar, se resalta el abordaje de la revelación y la aceptación de la os desde una variable cultural, lo que resulta pertinente debido a las pocas investigaciones al respecto a nivel mundial y a los relatos de los hijos participantes, que permitieron evidenciar que las situaciones de estigma y discriminación continúan latentes como un temor de la expresión de su sexualidad que también comparten las madres. En segundo lugar, se recomienda la utilización de técnicas interactivas como la línea del tiempo, herramienta que permitió mayor cercanía con los hijos y madres participantes para abordar temas que han movilizado emocionalmente a cada familia, como lo es el proceso de revelación y aceptación de la orientación sexual de un hijo.

En tercer lugar, se resaltan las principales temáticas encontradas en el estudio en relación con las características del proceso de revelación y aceptación de la os por parte del hijo: la autoaceptación, el proceso de descubrimiento de la identidad, los sentimientos asociados a la os, la figura materna como la primera persona a la cual se revela la os, los sentimientos de bienestar producto de la revelación, la sensación de embotellamiento; y en el caso de las madres, la ruptura de sistemas de creencias y actitudes ambivalentes frente a la homosexualidad de sus hijos. En cuando a las barreras, se evidenciaron la deshonra familiar, la endodiscriminación, el fenómeno de la doble discriminación (raza/etnia y os) y, como recurso por parte de las madres, la ruptura de los esquemas culturales para llevar a cabo la aceptación de la os de sus hijos.

En cuarto y último lugar, este estudio aporta al análisis interseccional de la relación e influencia de la raza, etnia y cultura en la visión y vivencia de la sexualidad humana, lo que evidencia que aspectos de 
la crianza, los estereotipos sociales, la racialización de la sexualidad y el fenómeno de doble vulneración son aspectos que obstaculizan los procesos de revelación y aceptación de la os, por lo que se generan necesidades de acompañamiento especiales que podrían ser abordadas por las instituciones educativas y por profesionales de la salud que trabajan con familias, a través de intervenciones que promuevan la reducción de prejuicios provenientes de la crianza y de creencias heteronormativas, de modo que permitan el reconocimiento de la interseccionalidad por raza/etnia, género y orientación sexual, y reduzcan la vulnerabilidad a distintos tipos de discriminación que de estas se derivan (Rubio, 2014).

Se reconocen, de esta manera, elementos clave para la intervención con familias afrocolombianas con hijos/as gais y lesbianas, como brindar conocimientos científicos sobre diversidad sexual y de géneros que favorezcan el bienestar de sus integrantes, ya que muchos de los temores y barreras de las madres se encuentran relacionados con la desinformación. Asimismo, apoyar la identificación de redes de apoyo, aspecto que fue mencionado por las dos familias como una ayuda que les habría gustado tener durante su proceso, en especial debido a sus contextos de vulnerabilidad. De igual forma, se considera fundamental trabajar en la comunicación familiar, como aspecto facilitador de la aceptación de la os y el enfrentamiento de situaciones de racismo y homofobia.

\section{Referencias}

Almario, Óscar; Lasso, Marixa; Cunin, Elisabeth; Urrea, Fernando; Langebaek, Carl y Chaves, Margarita. (2007). Aproximaciones a los estudios de raza y racismo de Colombia. Revista de Estudios Sociales, (27), 184-193. Recuperado de http://www.scielo.org.co/scielo.php?script = sci_arttext\&pid = S0123$885 \times 2007000200013$

Baiocco, Roberto; Fontanesi, Lilybeth; Santamaria, Federica; Loverno, Salvatore; Marasco, Barbara; Baumgartner, Emma; Willoughby, Brian y Laghi, Fiorenzo. (2014). Negative Parental Responses to Coming Out and Family Functioning in a Sample of Lesbian and Gay Young Adults. Journal of Child and Family Studies, 24, 1490-1500. https://doi.10.1007/s10826-014-9954-z. 
Blanco, Mercedes. (2015). Investigación narrativa: una forma de generación de conocimientos. Argumentos, 24(67), 135-156.

Bronfenbrener, Urie. (1987). La ecología del desarrollo humano. Barcelona: Paidós.

Carrion, Victor y Lock, James. (1997). The Coming Out process: Developmental Stages for Sexual Minority Youth. Clinical Child Psychology and Psychiatry, 2(3), 369-377.

Castellanos, Gabriela. (2008). Serialidad, dominación, performatividad: la construcción de identidades subordinadas y la aceptación de la subordinación. En Fernando Urrea, Mara Viveros y Peter Wade (Eds.), Raza, etnicidad y sexualidades: ciudadanía y multiculturalismo en América Latina. Bogotá: Centro de Estudios Sociales, Universidad Nacional de Colombia.

Ceballos-Fernández, Marta. (2014). Identidad homosexual y contexto familiar heteroparental: implicaciones educativas para la subversión social. Revista Latinoamericana de Ciencias Sociales, Niñez y Juventud, 12(2), 643-658.

Colombia Diversa. (2018). Entretejiéndonos. Herramientas para la defensa de los derechos económicos, sociales y culturales de la población afro e indígena LGBT en Colombia. Recuperado de https://colombiadiversa.org/colombiadiversa2016/wp-content/uploads/2019/02/entretejiendonos_digital.pdf

Comisión Interamericana de Derechos Humanos [CIDH]. (2015). Violencia contra personas LGBTI. Recuperado de http://www.oas.org/es/cidh/informes/ pdfs/violenciapersonaslgbti.pdf

Congreso de la República. Ley 70 de 1993. Por la cual se desarrolla el artículo transitorio 55 de la Constitución Política. Agosto 31 de 1993. DO N. ${ }^{\circ} 41.013$. Recuperado de https://www.minagricultura.gov.co/Normatividad/Leyes/ Ley \% 2070\%20de\%201993.pdf

Congreso de la República. Ley 1090 de 2006. Por la cual se reglamenta el ejercicio de la profesión de Psicología, se dicta el Código Deontológico y Bioético y otras disposiciones. Septiembre 6 de 2006. DO N. ${ }^{\circ} 46.383$. Recuperado de http://www.secretariasenado.gov.co/senado/basedoc/ley_1090_2006.html

Consejo Nacional de Política Económica y Social. (23 de mayo de 2002). Política para la población afrocolombiana. Recuperado de https://www.minagricultura.gov.co/Normatividad/Conpes/Conpes \% 203169\%20de\% 202012.pdf 
Curiel, Ochy. (2014). Género, raza, sexualidad. Debates contemporáneos. Recuperado de http://bdigital.unal.edu.co/39755/

Departamento Administrativo Nacional de Estadística [Dane]. (2019). Población negra, afrocolombiana, raizal y palenquera. Resultados del Censo Nacional de Población y Vivienda 2018. Recuperado de: https://www.dane.gov.co/files/investigaciones/boletines/grupos-etnicos/presentacion-grupos-etnicospoblacion-NARP-2019.pdf

Gil, Franklin. (2008). Racismo, homofobia y sexismo. Reflexiones teóricas y políticas sobre interseccionalidad. En Fernando Urrea, Mara Viveros y Peter Wade (Eds.), Raza, etnicidad y sexualidades. Ciudadanía y multiculturalismo en América Latina. Bogotá: Centro de Estudios Sociales, Universidad Nacional de Colombia.

González, Andrea. (2016). Agenciamientos micropolíticos de las disidencias sexo-genéricas en la Ciudad de México (Tesis de maestría). El Colegio de México, México, DF.

Hernández, Ángela. (2017). Familia, ciclo vital y psicoterapia sistémica breve. Bogotá, D. C.: Editorial El Búho.

Isaza-Camargo, Maricel. (2018). La experiencia histórica de las sexualidades disidentes en el mundo obrero. El caso del sindicato de las empresas municipales de Cali —Sintraemcali- 1980 al 2016 (Tesis de pregrado). Universidad del Valle, Cali, Colombia.

Jiménez, Monique; Borrero, Néstor y Nazario, Juan. (2011). Adolescentes gays y lesbianas en Puerto Rico: procesos, efectos y estrategias. Revista Puertorriqueña de Psicología, 22(1), 147-173.

Libson, Micaela. (2012). Parentalidades gays y lesbianas: varones y mujeres en familias no heteronormativas. Revista de Estudios de Género. La Ventana, 4(35), 292-321.

Lujan, Isabel y Tamarit, Ana. (2012). Dinámica familiar ante la revelación de la orientación homosexual de los hijos/as. International Journal of Developmental and Educational Psychology, 3(1), 301-308.

Macionis, John y Plummer, Ken. (2007). Sociología. Madrid: Pearson Educación, S.A. 
Martínez-Salgado, Carolina. (2012). El muestreo en investigación cualitativa. Principios básicos y algunas controversias. Ciência y Saúde Coletiva, 17(3), 613-619.

Mieles, María; Tonon, Graciela y Alvarado, Sara. (2012). Investigación cualitativa: el análisis temático para el tratamiento de la información desde el enfoque de la fenomenología social. Universitas Humanística, (74), 195-225.

Ministerio de Salud. Resolución 8439 de 1993. Por la cual se establecen las normas científicas, técnicas y administrativas para la investigación en salud. Octubre 4 de 1993. Recuperado de https://www.minsalud.gov.co/sites/rid/ Lists/BibliotecaDigital/RIDE/DE/DIJ/RESOLUCION-8430-DE-1993.PDF

Ministerio de Salud y Profamilia. (2015). Encuesta Nacional de Demografía y Salud. Recuperado de https://profamilia.org.co/wp-content/ uploads/2018/12/ENDS-TOMO-I.pdf

Ministerio de Salud y Protección Social. (2015). Desigualdades sociales en salud en Colombia. Informe Nacional. Recuperado de https://www.ins.gov.co/ Direcciones/ONS/Informes/6.\%20Desigualdades \%20sociales.pdf

Mogrovejo, María Norma. (2008). Diversidad sexual, un concepto problemático. Trabajo Social, (18), 62-71. Recuperado de http://www.revistas.unam.mx/ index.php/ents/article/view/19577/18571

Moncada-Santos, Margarita y Fontelo-Danta, Lissell. (2018). La construcción cultural de la sexualidad en adolescentes. Santiago, 146, 331-347.

Orcasita, Linda; Sevilla, Teresita María; Acevedo-Velasco, Victoria Eugenia; Montenegro, José Luis; Tamayo, María Camila y Rueda-Toro, Juan Sebastián. (2020). Apoyo social familiar para el bienestar de hijos gays e hijas lesbianas. Revista Latinoamericana de Ciencias Sociales, Niñez y Juventud, 18(2), 1- 23. http://dx.doi.org/10.11600/1692715x.18205

Ortiz-Hernández, Luis. (2004). La opresión de minorías sexuales desde la inequidad de género. Política y cultura, (22), 161-182.

Programas de las Naciones Unidas para el Desarrollo [PNUD]. (2011). Afrocolombianos - Sus territorios y condiciones de vida. Recuperado de https://www. co.undp.org/content/colombia/es/home/library/human_development/ afrocolombianos---sus-territorios-y-condiciones-de-vida.html 
Rodríguez, Jesús. (2005). Definición y concepto de la no discriminación. El cotidiano, 21(134), 23-29. Recuperado de http://puntogenero.inmujeres.gob. $\mathrm{mx} / \mathrm{madig} /$ discriminacion/docs/ArticuloJesusRodriguez.pdf

Rothman, Emily; Sullivan, Mairead; Keyes, Susan y Boehmer, Ulrike. (2012). Parents' Supportive Reactions to Sexual Orientation Disclosure Associated with Better Health: Results from a Population- Based Survey of LGB Adults in Massachusetts. J Homosex, 59(2), 186-200.

Rubio, Aimar. (2014). Orientación sexual, violencia y derechos humanos en África. El caso de Sudáfrica (Tesis Doctoral). Universidad del País Vasco, Bilbao, España.

Rueda, Miguel. (2015). El significado del prejuicio sexual en las trayectorias de la identidad con la orientación sexual homosexual y bisexual (Tesis doctoral). Universidad de los Andes, Bogotá, Colombia. Recuperado de https:// repositorio.uniandes.edu.co/handle/1992/7713

Salgado. Ana. (2007). Investigación cualitativa: diseños, evaluación del rigor metodológico y retos. Liberabit, 13(13), 71-78.

Salinas, Héctor Miguel. (2010). Políticas de disidencia sexual en América Latina. Sujetos sociales, gobierno y mercado en México, Bogotá y Buenos Aires. México, DF: Ediciones y Gráficos Eón.

Silva, Benjamín. (2018). Efectos en el afrontamiento y soporte social ante la revelación de la homosexualidad a la familia: estudio comparativo en gays y lesbianas. Psicogente, 21(40), 321-336.

Stake, Robert. (2007). Investigación con estudio de casos. Madrid: Ediciones Morata.

Svab, Alenka. (2016). Narratives of coming out to parents: results of replicating a sociological study on the everyday life of gays and lesbians in Slovenia (2014-2015). Teorija in praksa, 53(6), 1344-1355.

Taylor, Steven y Bogdan, Robert. (1992). La entrevista en profundidad. En Introducción a los métodos cualitativos en investigación: la búsqueda de significados. Barcelona: Paidós Ibérica. 
Troutman, Omar y Evans, Kathy. (2014). A Psychoeducational Group for Parents of Lesbian, Gay, and Bisexual Adolescents. Journal of School Counseling, 12(18). Recuperado de http://jsc.montana.edu/articles/v12n18.pdf

Urrea, Fernando; Reyes, José y Botero, Waldor. (2008). Tensiones en la construcción de identidades de hombres negros homosexuales en Cali. En Fernando Urrea, Mara Viveros y Peter Wade (Eds.), Raza, etnicidad y sexualidades: ciudadanía y multiculturalismo en América Latina. Bogotá: Centro de Estudios Sociales, Universidad Nacional de Colombia..

Vargas, Elvia; Ripoll, Karen; Carrillo, Sonia; Rueda, Miguel y Castro, Jhon. (2011). Experiencias familiares de madres y padres con orientaciones sexuales diversas, aportes de la investigación. Colombia: Ediciones Uniandes.

Vargas-Trujillo, Elvia; Villalobos, Sandra; Trevisi, Giustina; González, Felipe y García Paula. (2003). Variables psicosociales asociadas con el grado de aceptación de la OS no heterosexual. Psicología desde el Caribe, (12), 39-51.

Willoughby, Brian; Malik, Neena y Lindahl, Kristin. (2006). Parental Reactions to Their Sons' Sexual Orientation Disclosures: The Roles of Family Cohesion, Adaptability, and Parenting Style. Psychology of Men \& Masculinity, 7(1), 14-26.

Zambrano, Christian; Ceballos, Ana y Ojeda, Dayra. (2017). Reconocimiento de la orientación homosexual. Revista Psicoespacios, 11(19), 77-93. https://doi. org/10.25057/21452776.940 